\title{
Subject Index to Abstracts
}

Abortion 767

Absorption, gastrointestinal 715

Absorption, passive 706

Abstinence 1401

Abuse 24, 28, 37, 105

Abuse, sexual 1085

Abuse, substance 62

Acanthosis 1217

Accidents 511, 518

Accretion 300

Acetaldehyde 777

Acetaminophen 282, 297, 381, 390

Acetylcholine 277

Acetyl-CoA 1200

$N$-Acetyl- $\beta$-D-glucosaminidase 411

$N$-Acetylglucosaminylphosphotransferase 745

Acetyl salicylic acid 352

Achievement testing 789

Achondroplasia 784, 1255, 1715, 1836

Acid 657

$\alpha$, -Acid glycoprotein 1136,1466

Acidification 1639

Acidity 1513

Acidosis 249, 630, 885, 1067, 1415,1574

Acid secretagogues 275

Acid secretion 625

Aciduria, argininosuccinic 1341

Acinetobacter glutaminase-asparginose 635

Acini 727

Acne 14

Acoustic method 731

Acoustic reflexes 810

Acquired immune deficiency syndrome $121,550,553,868,886$ $944,948,949,954,968,970$, $972,975,984,994,995,999$, $1008,1009,1010,1013,1014$, $1015,1016,1046,1053,1090$, 1096

Acquired monosaccharide intolerance 665

Actin microfilament 725

Actinomycin D 873

Acute lymphocytic leukemia 636, $849,865,887,890,916,922$

Acute nonlymphoblastic leukemia 862, 911

Acute nonlymphocytic leukemia 848

Acyclovir 1119

Acyl-CoA 755, 1227, 1236

Acyldehydrogenase 1236

Adenine nucleotide 1180

Adenine nucleotide translocase 26

Adenoid 1064

Adenoidectomy 797
Adenosine 215, 389

Adenosine deaminase deficiency 888

Adenosine triphosphate 839

Adenovirus 542, 1044, 1073, 1133

Adenylate kinase 1648

Adipocytes 441

Adipose tissue 231, 691

Adiposity 673

Admissions 825

Adolescence 1, 3, 4, 9, 10, 12, 16, $19,22,24,25$

Adrenal 14, 315, 405, 413, 446

Adrenal hormones 18

Adrenal medulla 270

Adrenarche 413, 502

Adrenarche, premature 495

Adrenergic agents 372, 1869

Adrenergic mechanism 287

$\alpha$-Adrenoceptor 1590

$\beta$-Adrenoceptor 321, 370, 1735

Adrenoleukodystrophy 1669

$\alpha_{2}$-Adrenoreceptors 296a

Adult respiratory distress syndrome, 1695, 1749

Aerobic 115

Aerosol therapy 1795,1847

Aganglionosis 622

Agglutinin 911

Aggression 9

Airway obstruction 1751, 1810, 1816

Airway pressure 1774,1780

Airway reactivity 1798

Alanine 450, 1242

Albumin 912, 1053, 1195, 1458

Alcohol 5, 303, 352, 355, 540, 1257,1261

Aldolase 928

Aldosterone 1633, 1646

Alkaline phosphatase 703, 1052

Allergy 633,1375

Allergy, atopic 961

Allotype 1079

Alloxan 1182

Aluminum 1634

Aiveolar capillary 1768

Alveolar overdistention 1778

Alveolar pressure 1772, 1773

Alveolar type II cell 304

Amaurosis 1669

Ambulatory care 1759

Amenorrhea 473, 479

Amikacin 353

Amiloride 729, 1573

Amino acid 423, 559, 573, 581, $625,626,629,660,693,694$ $730,771,1185,1231,1577$, 1586, 1617

Aminoaciduria 1585

Amniocentesis 774, 776

Aminodarone 351
Aminoglycoside 342,1140

Aminonucleoside nephrosis 1598

Aminophylline 1371, 1853

Aminopyrine 388

Ammonia 1397, 1707

Amnesia 825

Amniotic fluid 229, 618, 779, 1304,1535

Amniotic leak, prolonged 1739

Amoxicillin 819

Amphotericin 900, 1350, 1369

Ampicillin 1114, 1147

Amrinone 175, 345

Androgen 14, 449, 465, 1217

Anemia $20,140,143,500,545$, $816,835,846,888,896,904$, 1480

Anesthesia 1190, 1444

Angiocardiography 210

Angiography 202, 1591

Angioplasty, balloon 186

Angiotensin II 368

Ànorectoplasty 591

Anorexia 1166

Anorexia nervosa 180

Anthropometric variable 498, 722

Antiarrythmia 351

Antibiotic 379, 892, 1057, 1069 , 1110,1140

Antibody 937, 940, 942, 952, 958 , $972,985,995,999,614,671$, $753,758,844,850,852,897$, $1023,1024,1045,1049,1078$, $1100,1141,1151,1157,1270$ 1314,1650

Antidiuretic hormone 827

Antigen $280,614,707,945,957$ 1000,1093

Antihistamine 1566

Antioxidant 669

Antipyretic 1583

Antithrombin III 878, 912

$\alpha_{1}$-Antitrypsin $609,714,720,1844$

Antiviral 1084

Anxiety 42

Aorta 157

Apgar score 1719

Aplasia, pituitary 426

Apnea 34, 38, 263, 387, 561, 565, $634,797,1280,1286,1309$ $1310,1362,1372,1385,1394$, $1430,1432,1442,1517,1518$, $1520,1547,1552,1672,1737$, $1738,1785,1805,1807,1809$, $1810,1836,1842,1843,1846$, $1849,1853,1862,1869$

Apolipoprotein 749, 955

Apoprotein 1880

Appendectomy 512

Appendicitis 512

Appointment keeping 12

Arachidonate synthesis 1325
Arachidonic acid 295, 925, 1125, 1139,1254

Arginine 134

Arginine vasopressin 234, 235, 249

Argininosuccinicaciduria 781

Argininosuccinate 742, 1166

Arrhythmia 179, 195, 222, 223, $377,857,913,1796$

Arteriohepatic dysplasia 1642

Artery 1753

Artery, carotid 201

Artery, mesenteric 372

Artery, pulmonary $186,217,328$. 510

Arthritis 1167

Artificial endocrine pancreas 1211

Arylsulfatase B 758

Asbestos 539

Ascites 199

Ascorbate 394

Asphyxia 78, 249, 261, 315, 509 $912,1363,1366,1402,1440$ $1478,1537,1562,1565,1606$, $1627,1673,1678,1680,1686$, $1697,1708,1710,1722,1729$

Aspirin 692, 1207

Asthma 788, 1742, 1795, 1796, 1799,1804

Ataxia 702

Atrophy, gyrate 782

Attachment 62

Attention deficit 50, 70, 71, 113

Auditory brainstem response $1276,1286,1382,1445,1683$, $1684,1690,1691,1706,1725$

Auditory function 342

Auditory responses 242

Autoimmunity $846,837,938,946$, $950,969,996,1006$

Autopsy 1426

Autoregulation 269, 276

Atherosclerosis 557

Atopy 1375

Atresia 1264

Atresia, biliary 612,730

Atresia, pulmonary 136, 158, 202

Atriopeptin 1581

Atrioventricular valve insufficiency 206

Atrium 191, 207, 224

Bacitracin 1056

Bacteremia 879, 1043, 1057, 1059, $1095,1118,1135$

Bacteria 732, 1152

Bacteria, overgrowth 650

Bacterial disease 1061

Bactericidal activity 615,1069

Bacteroides fragilis 1108

Barium enema 713

Barotrauma 1370 
Barrett's esophagus 578, 616

Basement membranes 1768

Basolateral membrane 1610

Basophil 953

Bayley scores $65,67,85,89$

B cells $940,944,950,975,989$, $1002,1004,1202$

Behavior 25, 30, 31, 73, 100, 116, 128,1668

Behavior, maternal 61

Behavior, parental 7

Benzoate 746, 1206

Benzocaine 392

Benzyl alcohol 385, 386, 1322, 1474

Beriberi 1731

Betamethasone 1371, 1845

Bicarbonate metabolism 1214

Bicuculline 332

Bile $605,612,696,1190$

Bile acid 573, 587, 679

Bile acid, metabolism 627

Bile salts 564, 706

Biliary tract 726

Bilirubin 236, 237, 294, 326, 605 , $640,641,841,1053,1190,1276$, $1305,1311,1312,1329,1400$, $1417,1445,1450,1467,1487$, $1495,1514,1523,1524,1542$, $1553,1563,1570$

Bilirubinometry, transcutaneous 236,1400

Bioavailability $369,598,678$

Biopsy 1626, 1654

Biopterin deficiency 1209

Biostater 1211

Biotin 951, 1221, 1730

Biotinidase 617, 1730

Birth defect 83, 84, 205

Birthweight, maternal 532

Bladder 1638

Bleeding time $867,869,925,1506$

Blindness 123, 127

Blood 839, 861, 906, 955, 960, 983, 1042, 1055, 1057, 1322

Blood-brain barrier 1553, 1703 , 1722

Blood count 908

Blood pressure 156, 183, 184, $1308,1336,1352,1353,1449$, $1560,1561,1655$

Blood viscosity 1486

Body volume 731

Bonding 91

Bone 486, 1538, 1593, 1625, 1641

Bone disease 1634

Bone infarction 909

Bone marrow $833,860,864,872$ $876,896,917,929,932,952$ $959,967,974,993,1556$

Bone mineralization 579, 611, 645

Botulism 577

Bradycardia 54, 1518

Brain 247, 269, 280, 307, 326, 337, $362,372,373,407,434,677$ $1053,1175,1182,1245,1294$, $1311,1312,1326,1366,1450$, $1670,1678,1689,1694,1698$, 1722

Brazelton scale $73,74,112,1725$
Breath hydrogen 32

Breathing $100,324,325,389$, $1439,1519,1521,1549,1738$, $1784,1814,1817,1838$

Breath test 388

Breech 1321

Bronchial reactivity 1756

Bronchiolitis 1865

Bronchodilator 1795, 1796, 1799

Bronchopulmonary dysplasia 183 , $384,1358,1421,1432,1465$, $1515,1766,1775,1783,1797$, $1803,1815,1817,1821,1829$, 1844

Broviac catheter chemotherapy 1120

Broviac catheter infections 1120

Broviac catheter neutropenia 1120

Brush-border membrane 569, $1185,1585,1586,1597,1620$, 1621

Bucindolol 178

Burns 28, 36, 1124

Bypass, cardiopulmonary 350

Bypassing activity 856

\section{$\mathrm{C} 3 \mathrm{NeF} 1650$}

C5a des Arg 1756

Caffeine 324, 325, 361, 1384, 1671,1672

Calcification 1641

Calcitonin 486, 709, 1537

Calcium 144, 169, 187, 311, 319, 336, 346, 499, 579, 598, 660, $859,924,1194,1219,1233$, $1234,1301,1534,1538,1543$, $1562,1597,1604$

Calcium, parenteral 1534

Calcium gluconate therapy 1601

Calciuria 1601

Calories 673, 721, 1401

Calorimetry 693

Cancer 635, 845, 870, 891, 898, 1081,1120

Candida 945, 1038

Candida albicans 1612

Candida antigen 1101

Candidiasis 1101, 1350, 1369

Capnograph 1856

Captopril 1662

Carbamazepine 897

Carbamoyl phosphate synthetase 1203, 1216

Carbenicillin 1081

Carbohydrate $637,642,651,686$, 701, 1226

Carbon dioxide 1811

Carbon dioxide, alveolar-arterial 1867,1868

Carbon dioxide, transcutaneous 793,1748

Carbon dioxide production 1757

Carbon dioxide response 1829 , 1846

Carbon tetrachloride 496

Carboxylase 951

Cardiorespiratory arrest 513

Care, convalescent 79

Care, day 529

Care, home 1421
Care, level of 806,814

Care, medical 798

Care, prenatal 1476

Care, postoperative 1777

Care, well-child 69

Caregiver 41

Carnitine 698, 743; 755, 1200 1227, 1231, 1236, 1663, 1707

Carnitine deficiency 1210

Carotene 669, 889, 1436

Carotid bodies 1869

Carrier detection 741

Cartilage 279

Case definition 1009

Castration 251

Catecholamine 228, 261, 270, 288, 315,1403

Catheter, arterial 1307, 1308

Catheter, bulb vs deLee 1320

Catheter, peripheral artery 1308

Catheter, suction 1825

Catheter, umbilical 1353, 1356, 1530

Catheterization gradients 159

Cavum vergae 1266

Cefotaxime 1036

Cefoxitin 1062

Ceftazidime 1081

Ceftriaxone $333,1040,1117$

Celiac disease 562, 563

Central hypoventilation syndrome 759

Central nervous system 887,916 , 1350,1709

Cephalosporins 339, 1050

Cephalothin 108

Cereal 512

Cereal prolamins 563

Cerebral palsy 740

Cerebrospinal fluid 815,887 , Coagulation 867, 885, 913,998 $1117,1675,1680$

Ceruloplasmin 601,717

Cesarean section 1342

Chediak-Higashi syndrome 899 936, 986

Chelation 710,901

Chemiluminescence 1003

Chemoprophylaxis 916

Chemoreceptors $325,1483,1823$. 1824

Chemotaxis 398, 615, 883, 934, $1011,1281,1282$

Chemotherapy 849, 862, 895, 922

Chest wall 1394

Child abuse 518,812

Chileans 1399

Chlamydia 8, 1086, 1103

Chloramphenicol 335, 1067, 1080 1239

Chloride 612, 1176

Cholecystectomy 893

Cholestasis 587, 620, 627, 680, $702,725,1169,1642$

Cholesterol 240, 1181, 1436

Chorioanmionitis 1373,1478

Choroid plexus $865,1293,1692$

Chromosome $739,750,760,767$

$774,786,896,1032$

Chromosome 8748

Chromosome, sex 776
Chromosome, X 770, 786

Chromosome abnormality 520, 772, 776, 779

Chronic granulomatous disease 876,1712

Chronic lung disease 1776

Chronopharmacology 505

Chylomicron 749

Cimetidine 357, 656

Circadian rhythms 291, 292, 1848

Circulation, cerebral 215, 276, $307,332,1275,1279,1287$, $1398,1407,1415,1416,1435$, $1449,1453,1526,1547,1548$, $1686,1687,1694,1699,1704$, 1716,1726

Circulation enterohepatic 706

Circulation, gastrointestinal 711

Circulation, heart 201, 211, 1290

Circulation, kidney 1571,1624

Circulation, organ 170, 1813

Circulation, peripheral 190

Circulation, pulmonary 141,142 , $338,395,1297,1509,1734$, 1774

Circulation, regional 160, 199 , $200,734,1360$

Circumcision 46, 796

Circumference, mid-arm/head 603

Citrulline 267, 1665

Citrullinemia 742

Classroom performance 1346

Cleft lip 483, 1258

Cleft palate 483, 1123, 1254

Cleft, oro-facial 1264

Clonidine 445, 485

Cloning 422, 832, 866

Clonogenic 870

Coarctation 147,219

Cobalamin 764,1220

Cognition 102, 103, 107, 120, 125, 1684

Colchicine 1618

Colitis 567, 583, 674

Collagen $606,784,785,1852$

Colloid osmotic pressure 170,210

Colon 577

Coma 619

Committee 57

Complement 913, 939, 943, 1021

Complex 1766

Compliance 10, 12, 17, 39, 1787 , 1872

Computer 58, 70, 76, 95, 97, 135, $316,534,1459$

Congenital adrenal hyperplasia $427,435,460,464,495$

Constipation 653,713

Contacts 1863

Contraceptives, oral 8

Continuous positive airway pressure 1430

Contractility 132,193

Coordination 56

Copper 716, 717, 1679

Cornstarch 1179,1183

Coronary artery aneurysm 220

Coronary artery disease 151 
Coronary fistulae 158

Coronavirus-like 1108

Cor pulmonale 1797

Corrected age 67

Cortical 1666

Corticosensory 56

Corticosteroid 264, 583, 1779, 1803

Corticosterone 489

Cortisol 21

Cortisol binding globulin 455

Costs 43, 92, 101, 106, 1468, 1759

Cotinine 802

Coxsackie B 508, 998

C-reactive protein 987, 1092, $1136,1466,1603$

Creatine 782, 933

Creatine kinase 1729

Creatinine 378, 1277, 1328, 1572, 1601

Crohn's disease 606, 609, 639

Cross-culture 69

Croup 1026

Cry, abnormalities 40

Cry analysis 40

Crying 32, 72, 599

Crying baby syndrome 599

Cryopreservation 1404

Cryptophthalmos 1259

Cryptosporidium 1154

Cushing's syndrome 493

Cutis laxa 1251

Cutis verticis gyrata 1252

Cyanosis 129, 131, 209

Cycloheximide 302

Cyclohydrolase 765

Cyclooxygenase 1828

Cyclosporine 969, 1657

Cyclosporin-A 860, 927

Cysteine 630

Cystic disease 1256

Cystic fibrosis $335,548,555,556$,

$585,623,625,644,649,695$

$729,743,1043,1176,1614$

$1835,1881,1882$

Cystine transport 250, 1592

Cystinosis 302, 403, 751, 752, 783

Cytochrome oxidase 756

Cytomegalovirus 529, 936, 1034 1037, 1045, 1091, 1096, 1097 $1104,1111,1138,1143,1144$, $1146,1149,1351,1404,1515$, 1727

Cytoprotection 656

Cytoskeleton 310

Cytotoxicity 947, 950, 960, 978, $979,981,986$

Danazol 856, 867

Dapsone 1090

Database 95, 534

Deafness 1028

Death, sudden 195, 196

3-Deazoadenosine 1850

Decision making 790, 796

Defects 167,1564

Deferroxamine 1584

Degeneration 1669

Degranulation 872
Dehydroepiandrosterone 497

Delinquency 20

Deliver 109, 1005

$\mathrm{N}$-Demethylase 388

Densitometry 700

Depressant 1132

Depression 7

Dermatomyositis 998

Dermatopharmacology 390

Detection 72

Development, abnormalities 121

Development, bile 696

Development, brain 733

Development, cognitive 29, 126

Development, early 312

Development, gestational 229

Development, kidney 250

Development, lung 1735, 1807, 1840

Development, mental 31, 62, 117

Development, mitochondrial 260

Development, motor 29, 107

Development, neuro 27

Development, neuromotor 126

Development, outcome $38,45,60$, $64,99,122,807$

Development, reactions 11

Development, sexual 456

Development, small intestine 707

Dexamethasone $71,434,1340$, $1358,1536,1771$

Dextrostix 1355

Diabetes 13, 23, 411, 415, 416, $443,481,488,753,801,1178$, $1182,1195,1202,1204,1211$, $1215,1223,1228,1234,1243$ $1337,1573,1760$

Diabetes, maternal 268, 497, 677, $969,1231,1283,1337,1470$ 1502,1559

Diagnosis, prenatal 419, 460, 760, 776, 779, 790

Dialysis $1580,1593,1609,1622$, $1628,1649,1658,1663$

Diaphragm 1394, 1736, 1839

Diarrhea 379, 514, 564, 610, 642 . $651,686,1044,1073,1107$ 1133,1154

Diazoxide 1225

Dicarboxylic aciduria 1165, 1200, 1724

Dichloroacetate 1206

Diet $688,1215,1577$

Diet, maternal 568, 705

Diet control 17

DiGeorge syndrome 959, 1006

Digoxin 163, 351

Dihydrotestosterone 304,305

1,25-Dihydroxyvitamin D (see Vitamin D) 709

1,25-Dihydroxyvitamin $\mathrm{D}_{3} \quad 983$, 1174

Dilatation, esophageal 668

Diltiazem 346

Dinamap 1560, 1561

Diphenhydramine 395

2,3-Diphosphoglycerate 839,1235

Diptheria 937, 945, 1162

Disabilities 27, 106

Disaturated phosphatidylcholine
298,1551

Discharge, post-term 1405

Discipline 24

Dish soap 818

Diuresis 301

Diuretics 317

Diversion 1638

DNA breakage 739

Dobutamine 218

L-Dopa 762

Dopamine 228, 232, 315, 356, $805,1589,1624,1685$

Doppler 132, 139, 157, 159, 164, $172,177,191,201,206,219$ $1278,1435,1678,1699$

Down syndrome $767,836,1264$ 1269

DPT $554,791,1039$

Drug 5, 37, 1407

Drug, abuse 135

Drug, toxicity $318,341,3851067$

Drug, use 396, 1261

Drug abuse, pregnancy 1017

Drug addiction 329, 330, 348, 498

Drug addiction, infant 1605

Drug addiction, maternal 722 , 948, 1013, 1017

Drug displacement 326

Drug monitoring 331

Drug resistance 832,1863

Duchenne muscular dystrophy 774,1712

Ductus arteriosus 139, 188, 213, $219,391,1316,1424,1443$, 1511,1526

Dwarfism 462, 1249

Dysautonomia 1636

Dysgammaglobulinemia 874

Dysgenesis, gonadal 768

Dysgenesis, reticular 965

Dysgenesis, testicular 504

Dysgraphia 70

Dysmorphism, 1224, 1245

Dysplasia 1249

Dysplasia, islet cell 643

Earphone 1691

Ebstein's anomaly 129

Echinocytosis 855

Echocardiography 129, 133, 135 154, 177, 203, 205, 207, 212, $213,220,224,225,1145,1477$ 1530

Economics 796, 1451

Echovirus 11531

E. cloacae 1050

Edema 1789

Edema, lung 1303, 1458

Education $811,821,828$

Education, continuing medical 806

Education, patient 88

Education, pediatric 813

Education, residency 823

Elastase 1851

Electrocardiography 148, 203

Electrodiagnosis 1605

Electromyogram 1736

Electropherotype 1065

Electrophysiology 146, 147, 149,
$153,222,223,277,377,729$

Embryo 152, 1271

Embryology 1262

Emesis 818

Emotional disturbance 111

Emphysema, pulmonary interstitial 1380

Enamel, preterm 94

Encephalitis 963

Encephalopathy 730, 1686, 1717

Encopresis 653

Endocardial cushion defect 225

Endocarditis, experimental 1099

Endocrine 230

$\beta$-Endorphin 367, 387, 491, 815, 1680,1717

Endoscopic retrograde cholangiopancreatography 726

Endothelial cells 266

Endotoxin 285, 299, 1042, 1140 , 1160

Endotracheal tubes 1347,1792

End-tidal $\mathrm{CO}_{2}$ 1866, 1867, 1868

Endurance training 479

Energy expenditure 648, 1214, 1460

Enkephalin 270

Enolase 928

Enteric protein loss 609

Enteritis 477

Enterococci 1099

Enterocolitis 600, 736

Enteroinsular axis 667

Enterovirus 508, 531, 808, 1134, 1145

Environment 525

Enzyme 298, 763, 1338, 1771

Enzyme, restriction 1048

Enzyme, gastrointestinal 618

Enzyme replacement, pancreatic 335

Eosinophils 918

Epidemiology 516, 523, 530, 550, 898, 1009

Epidermal growth factor 262, 296 , $476,484,690,715,1403,1413$, $1760,1779,1845$

Epidural narcotics 1777

Epilepsy 10, 336

Epinephrine 228, 289, 315, 328, $405,805,1449,1755$

Epithelium 1826, 1827

Epstein-Barr virus 954, 1022, 1072

Erythrocyte 137, 594, 718, 855, $859,875,888,927,928,933$ $1235,1470,1712$

Erythrocyte-malondialdehyde assay 628

Erythrocyte progenitor cells 1186

Erythromycin-sulfisoxazole 819

Erythropheresis 192

Erythropoiesis 835

Erythropoietin 1523

Escherichia coli 773, 929, 1050 , $1051,1054,1059,1082,1100$ $1108,1131,1134,1368$

Eskimos, Alaskan 464, 769

Esophageal function 723

Esophagitis 578, 586, 633

Esophagus 668 
Ester glucuronide 380

Estrogen 435, 1735

Ethane and $N$-pentane 1782

Ethanol 341, 595, 777, 1248

Ethics 57, 792

Ethnicity 87, 543

Ethylmalonic-adipic aciduria 737

Evaluation 822

Evaluation, clerkship 795

Evaluation, residents 794

Evaluation, psychologic 681

Evoked responses 1698

Evolution 786

Exercise 115, 130, 131, 136, 150, $162,411,527,662$

Exercise training 13

Exocrine enzyme 272

Exocrine function 644,727

Exocytosis 1639

Exotoxin 1112

Expiration 1839, 1860

Extracorporeal membrane oxygenation 237

Extubation 1755

Face, abnormalities 1246

Factor VIII 266

Familial polyposis 674

Family 75, 82, 90

Fanconi syndrome 860,896

Fat 306, $608700,701,721,733$, 1231,1491

Fat, brown $296 a$

Fatigue 1736

Fatty acid 637, 708, 718, 1197 1324,1555

Fatty acid, essential 312

Fatty acid, free 240, 470, 1240, 1542

Fecal incontinence 591

Feeding 3, 238, 533, 1808

Feeding, breast $61,118,1674$

Feeding, nasogastric 712

Feeding intolerance 347

Fentanyl 350

Ferritin 20, 582, 853

Ferrochelatase 877

Fetal alcohol syndrome 1261

Fetal distress 1705

Fibroblast-pneumonocyte factor 1752,1831

Fibronectin 735, 962, 966, 1141, 1160,1775

Flavoprotein 737

Flavoprotein, electron transport 1188

Fluid balance 1688

Folate 1257

Follow-up $65,68,85,86,90,104$

Fomites 1351

Food, sensitization 1375

Formula 32, 590, 1428

Formula, soy-base 645

Fragile X 1714

Functional residual capacity 1481 , 1877

Fundoplication 604

Furosemide $317,331,380,1464$, $1538,1558,1564,1611,1627$ 1783
Furosemide glucuronide 380

Galactose 268, 1199

Gallstones 893

Gammaglobulin 846, 847, 1010, 1016,1020

Gardnerella vaginalis 1085

Gas, blood 92, 1417

Gas tension 229

Gastric acid 584, 625

Gastric aspirate 515

Gastric emptying 701, 712, 723

Gastric motility 711

Gastric wall 715

Gastrin 584

Gastroenteritis 1054

Gastroesophageal reflux 560,561 , $565,575,576,578,586,599$. $604,616,634,662,695,723$, 1821

Gastrointestinal bleeding 685,692

Gastrointestinal tract 672, 690, 710

Gaucher's disease 840

Gene 461, 462, 784, 832, 910, 915

Gene deletion 775

Genetic counseling 761

Genetics 898, 1007

Genitalia 1269

Gentamicin 316, 400, 1081, 1147 , 1423,1607

Gestational age 31, 231, 1291, 1790

Giardia lamblia 1060

Giardiasis 1060

Gliadin peptide 562

Global function 145

$\beta$-Globin 915

Glomerular basement membrane 1598

Glomerular filtration rate 1328 , $1569,1576,1655,1659,1660$

Glomerular injury 1636

Glomerulonephritis 1602, 1615 , 1630,1650

Glomerulopathy 1653

Glomerulosclerosis 1613, 1632

Glucagon $248,368,424,1173$, 1225

Glucoamylase 686

Glucocerebrosidase 840

Glucocorticoid 290, 457, 1284, $1371,1536,1582,1752$

Gluconeogenesis 256, 1191, 1201, 1237

Glucose 123, 246, 337, 365, 423, $424,574,581,651,654,667$, $676,693,1178,1179,1183$, $1192,1196,1339,1408,1454$, 1522

Glucose-6-phosphate dehydrogenase 928,1493

Glutamine 1198

Glutamyl-transpeptidase 1163, 1164,1185

Glutaric aciduria type II 737, 1188

Glutathione 297, 1878

Glycemia 1215

Glycerol 1237, 1675

Glycerolipid 1177
Glycine 1168

Glycogen $216,268,309,368$,

$1171,1190,1208,1238$

Glycogenolysis 252

Glycogenosis 477, 1179, 1183

Glycohemoglobin 1223, 1882

Glycolysis 290, 1235

Glycoprotein 728, 738, 935, 978, 1049

Glycosylation 416

Goblet cells 1851

Gonadal hormones 18

Gonadoblastoma 451, 1259

Gonadodysgenesis 1259

Gonadotropin 410, 453, 485

Gonadotropin releasing hormone 494

GP-138 deficiency syndrome 1012

Graft $v s$ host disease 932, 974

Granulocyte 883, 899

Granulocyte/macrophage 874

Granulopoiesis 892

Graves disease 1006

Grief 1381

Group A streptococcus 1047, 1056

Group B streptococcus 124, 374, $552,990,1024,1029,1070$, $1125,1141,1147,1313,1314$, $1343,1361,1377,1438,1446$

Growth, abnormalities 497

Growth, catch-up 66, 43!

Growth, delayed 440

Growth, impairment 320

Growth, intrauterine 300,303 , $306,341,361,365,476,760$, $1004,1173,1212,1399,1544$, 1559

Growth, perinatal 296

Growth, sexual 5

Growth, somatic 103

Growth factor I 428

Growth failure, diabetes-related 1204

Growth hormone 407, 414, 417, $418,424,431,441,443,445$, $448,457,461,462,472,480$ $482,483,487,490,492,503$ $566,1196,1255$

Growth hormone releasing factor $430,461,478,480,487$

Growth retardation 312

Guanethidine 370

Haemophilus influenza 549, 769 $819,929,937,1036,1040,1050$ $1057,1079,1080,1113,1114$ $1121,1126,1132,1135,1137$ $1150,1151,1153,1155,1162$, 1438

Handicap 68, 1539

Hazardous waste 525

Head CT scans 825

Head injury $36,825,827,880$

Hearing 33, 242, 1472, 1564

Heart 144, 146, 150, 160, 166$168,182,187,194,209,216$, $277,319,374,397,434,1132$, 1239,1543

Heart, abnormalities 212

Heart, arrhythmias 172
Heart, cardiomyopathy 134,221 , 303,1240

Heart, toxicity 386

Heart, transmedial rupture 186

Heart, univentricular 138

Heart block 1270

Heart catheterization 189, 205

Heart disease 149, 162, 171, 202, $206,901,1250$

Heart dysfunction 137, 180, 204, 214,218

Heart electrophysiology 181

Heart failure, congestive 178

Heart mass 180

Heart output 198, 1278, 1764, 1813

Heart rate 1849

Heat 1257

Height 26, 421

Helmholtz resonator 731

Hematocrit 906

Hematofluorometer 1305

Hematopoiesis 861, 899, 923, 967

Hematuria 1626, 1651

Hemodialysis 1584

Hemodynamics 131, 134, 142, $228,233,258,346,1290,1422$, $1579,1595,1762,1857$

Hemofiltration 1588

Hemoglobin 500

Hemoglobin, fecal 714

Hemoglobin F 837

Hemoglobin SS 837

Hemoglobinopathy 778

Hemolysis 666, 884, 933, 943

Hemolytic uremic syndrome 204 , $1644,1649,1662,1664$

Hemophilia 856, 857, 867, 868, $880,883,886,921,925,968$, $970,984,1022,1096$

Hemorheology 1334

Hemorrhage 89, 156, 734, 1479

Hemorrhage, intracranial 432, $1293,1318,1321,1396$

Hemorrhage, intraventricular 60 , $65,110,122,126,444,858$, $1275,1287,1342,1382,1386$ $1389,1392,1398,1411,1498$, $1501,1505,1506,1508,1528$, $1552,1540,1548,1668,1674$ $1677,1681,1688,1690,1693$, $1697,1704,1705,1708,1710$, $1711,1713,1718,1720,1721$, 1728

Hemorrhage, pulmonary 1306

Hemostasis 209, 1250

Heparan sulfate 1598

Heparin 273, 313, 1299, 1433

Hepatic clearance 1153

Hepatic lipase 313

Hepatitis 526, 535, 671, 719

Hepatocyte 287, 368, 697, 1191, 1208

Hepatotoxicity $282,297,1231$

Hereditary spherocytosis 1076

Hernia 1224, 1264

Hernia, diaphragmatic 1444, 1745

Herpes simplex 963, 979, 985, $1023,1035,1109,1119,1159$

Heterogeneity 781,894 
Hexokinase 928

Hexosamine-6-sulfate 744

Hexosaminidase 744, 747

Heymann nephritis 1618

High frequency oscillation 1512

Hirschsprung's disease 600, 622, 713

Hirsutism 446, 493, 1252

Hispanic 543, 1532

His-Purkinje 149

Histamine 328, 395, 953, 1875

Histiocytosis X 894

Histoplasma capsulatum 937,938

HLA antigens 753,1623

Homeostasis 967, 1226, 1383 , 1522

Homocystinuria 764

Hormones 281, 420, 1212, 1264 , 1593

Host defense 265, 932, 1019

Human $T$ cell leukemia virus 886 , $995,1013,1014$

Hyaline membrane disease 327 , $360,680,1295,1380,1455$, $1478,1511,1531,1545,1546$, $1787,1789,1873$

HY antigen 504,1273

Hybridoma 1024

Hydranencephaly 1668,1698

Hydrocephalus 1265, 1389, 1675, 1696, 1711

Hydrochlorothiazide 1573

Hydrocortisone 283, 505, 872

Hydrogen 632, 1296

Hydronephrosis 1578,1579

Hydrops 206, 1378

$\beta$-Hydroxybutyrate 733, 1723

26-Hydroxycholesterol 679

6-Hydroxydopamine 153

Hydroxyeicosatetraenoic acid 931 , 1228

21-Hydroxylase deficiency 422 , $427,460,464,495$

25-Hydroxylation 496

Hyperactivity 30,113

Hyperammonemia 1203, 1206, $1216,1341,1397$

Hyperandrogenemia 1217

Hyperbilirubinemia 40, 358, 1329 , $1333,1344,1445,1487,1493$ 1541

Hypercalciuria 1204, 1651

Hypercapnea 1398, 1800

Hypercarbia 1449, 1450

Hypercholesterolemia 1181

Hypercoagulable 1299

Hyperglucagonemia 248

Hyperglycemia 137, 216, 309, $1230,1238,1502$

Hyperinsulinemia 1238, 1502, 1554

Hyperinsulinism 1182, 1225

Hyperkalemia 1611

Hyperlipidemia 557

Hyperlipoproteinemia 749

Hyperosmolality 1311,1428

Hyperosmolar 953

Hyperoxia 1473, 1786, 1874

Hyperparathyroidism 1634

Hyperphenylalaninemia 754,765 ,
1209

Hyperphosphatasemia 703

Hyperphosphatasia 703

Hyperplasia, adrenal 420

Hyperprolactinemia 473

Hypertension 130, 165, 183, 184, $230,1307,1352,1353,1453$, $1591,1659,1661,1662,1689$

Hypertension, maternal 1327

Hypertension, pulmonary 35,225 , $239,345,376,402,1152,1340$, $1352,1364,1367,1434,1444$, 1802,1879

Hypertension, renovascular 1567

Hyperthermia, malignant 1263

Hyperthyroidism 150, 433, 484, 1006,1413

Hyperthyrotropinemia 458

Hypertriglyceridemia 1323, 1663

Hypertonicity 50

Hypertrophy 155

Hyperventilation 35, 1292, 1301, $1352,1364,1422,1820$

Hyperviscosity 1334, 1486, 1527

Hypoalaninemia 1212

Hypoaldosteronism 489

Hypoaminoacidemia 248

Hypobaria 328

Hypocalcemia 436, 499, 1537, 1543

Hypocemia 214

Hypogammaglobulinemia 974 , $990,1020,1285$

Hypoglycemia 214, 481, 1171, $1212,1522,1554$

Hyponatremia 489, 827, 1129 , 1580

Hypophosphatemia 1608

Hypopituitarism 406, 407, 421, $424,450,478,487,503$

Hypoplasia 224, 1006, 1268, 1753 , $1754,1767,1814$

Hypospadias 1251

Hypotension $507, \quad 680, \quad 1360$ $1454,1458,1628,1722$

Hypothalamus 408

Hypothermia 1471

Hypothyroidism 247, 287, 425,

$454,459,480,484,500,501$

Hypothyroxinemia 501

Hypoviscosity 1334

Hypovolemia 1360, 1587

Hypoxanthine phosphoribosyltransferase 740

Hypoxemia 140, 197, 233, 235, $269,711,1335,1454,1465$, $1697,1717,1804,1855$

Hypoxia 161, 174, 185, 222, 223, $234,239,257,261,301,328$ $491,1180,1244,1367,1376$ $1415,1492,1510,1547,1670$ $1686,1701,1717,1757,1758$, 1802

i (9q) 777

I cell 745,752

Icterometer 1495

Idiopathic infantile hypercalcemia 1189

Idiopathic thrombocytopenia pur- pura 847

I:E ratio 1801

IgA 650, 958, 982, 1018, 1025 , 1368,1568

IgD 976

IgE 961,1007

IgG $844,852,871,941,949,982$

$\operatorname{IgM} 1105$

Ileum 1638

Illness 75,820

Illumination, nursery 255

Imaging 166,167

Imipenem 339

Immigrants 519

Immune complexes 1010

Immune phenotype 894

Immune system 956

Immunity $683,944,960,962,963$, $966,985,997,1049,1107,1128$ $1138,1285,1812$

Immunization 510, 537, 554, 791, $940,941,1130,1142$

Immunodeficiency 553, 610, 886 921, 942, 950, 951, 952, 972, $993,999,1015,1017,1019$, 1020

Immunogenicity 1150

Immunoglobulins $610,647,842$, $884,943,975,976,988,1079$, $1088,1089,1128,1137,1151$, 1270

Immunohistochemistry 1109

Immunoperoxidase 914

Immunoregulation 986, 1001, $1002,1022,1028,1031,1143$

Immunosuppression 955, 964,

$983,1637,1657$

Impedance 141, 142

Inadvertent peep 1780

Indomethacin $327,356,391,1316$, $1318,1424,1477,1498,1499$, 1500,1511

Infection, 157, 526, 542, 664, 808, $811,816,843,845,929,935$, $948,963,982,1012,1061,1106$, $1124,1136,1148,1161,1351$, 1474,1600

Infectious mononucleosis 1072

Inflammatory bowel disease 567 , 583, 689

Influenza 544, 977, 1064, 1071, $1083,1084,1115,1158,1583$, 1833

Informed consent 46

Infrared 1288

Infusion, plasma 1664

Ingestion 36

Inotropes 1306

Inpatient 821,825

Insulin 13,208, 244, 247, 284, $423,424,431,434,488,498$, $506,667,722,801,1167,1173$, $1175,1182,1186,1192,1196$, $1202,1205,1208,1211,1217$, $1243,1283,1502,1752$

Insulin-like growth factor 1232

Intelligence $26,459,481,1459$, 1666

Interaction 55,817

Interferon 866, 956, 992
Interleukin 21027,1030

Interns 829

Interstitial nephritis 1630

Intervention for premature 1488

Intestine $283,285,290,566,602$, $606,632,652,660,682,719$ 1197,1198

Intoxication 595

Intracranial pressure 1457,1463

Intubation 1349

Inulin clearance 1328

Inversion 7750

Iodocyanopindolol 321

Iron $688,816,853,877,1584$

Iron, serum 558

Iron overload 902, 903

Irradiation 607,1288

Ischemia 158, 182, 1416, 1547, $1571,1595,1643,1686,1694$ 1703, 1717

Isoantibodies 838

Isochromosome 777

Isoniazid 369

Isoproterenol 152, 161, 197, 372

Isotretinoin 1265

Isovaleric acidemia 757, 1168, 1227

Isovaleryl-CoA dehydrogenase 757

Jaundice 358, 1400, 1495, 1523 , 1524,1725

Jejunal function 564

Jejunostomy 580

Jejunum 728

Jewish populations 747

Joint contractures 1268

Juvenile rheumatoid arthritis 1834

Kallikrein 1633

Kawasaki disease 151, 220, 352 , 1077,1156

Keratinocytes 390

Kernicterus 1312

Ketamine 194

6-Keto PGF 1 1a 1376

Ketogenesis 1191

Ketone 733

Ketonemia 1212, 1231, 1723

Kidney 301, 1538, 1571, 1587 , $1624,1625,1635,1645,1646$, 1648

Kidney, abnormality 1245

Kidney, function $232,378,1569$, 1599

Kidney, malformations 165

Kidney, parameters 356

Kidney disease $1256,1574,1582$, $1588,1609,1628$

Kidney failure $1425,1469,1568$, $1595,1604,1609,1619,1625$, $1636,1643,1660$

Kidney sodium handling 1614

Kidney tubule 250, 971, 1592 , $1611,1614,1640,1647$

Kidney vascular resistance 235

King syndrome 1263

Kinin 1633

Kinky hair 1679

Klebsiella pneumoniae 595, 1082 , 1120

Kupffer cell 697 